\title{
INFORMACIÓN, MULTIMEDIALIDAD Y PERSONALIZACIÓN EN LAS RADIOAPPS DE ESPAÑA Y PORTUGAL
}

\author{
Information, multimediality and customization in radioapps \\ of Spain and Portugal
}

Teresa Piñeiro-Otero

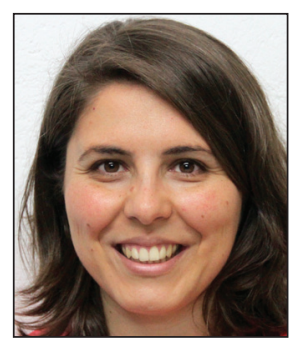

Teresa Piñeiro-Otero es doctora en comunicación por la Universidade de Vigo y profesora ayudante doctora en la Universidade da Coruña, donde imparte estrategias de comunicación multimedia y ambientación sonora y musical en el grado de comunicación audiovisual. Miembro del grupo de investigación Comunicación y Cultura Interactiva, ha orientado su investigación hacia las nuevas manifestaciones mediáticas en el contexto de la convergencia, con especial atención a la radio.

http://orcid.org/0000-0001-6414-2700

Universidade da Coruña, Facultad de Ciencias de la Comunicación Campus de Elviña. 15071 A Coruña, España teresa.pineiro@udc.es

\section{Resumen}

El consumo de la radio en los teléfonos inteligentes ha ampliado sus posibilidades comunicativas gracias a las características de esta plataforma de difusión. La concepción de los smartphones como pantallas donde convergen contenidos y comunicación, brinda a las radios la oportunidad de enriquecer su mensaje multimedia, apostar por nuevas fórmulas de distribución y avanzar en la personalización del medio. La conectividad de dichos terminales y su tecnología de geolocalización los dota de gran potencial para la información local, posibilitando una experiencia de consumo contextual. Se analizan las apps de las emisoras generalistas de España y Portugal, tanto desde la perspectiva de los contenidos como de las posibilidades para personalizar su acceso y consumo.

\section{Palabras clave}

Radioapps; Radio móvil; Radio online; Aplicaciones; Emisoras; Smartphones; Dispositivos móviles; Multimedialidad; Personalización, España; Portugal.

\section{Abstract}

The shift of radio listeners from radios to smartphones has expanded the communicative potential of the broadcasters due to the characteristics of this new distribution platform. The convergence of content and communication on the smartphone screen gives radio stations the opportunity to enrich their multimedia messages, explore new ways of distribution and achieve advances in customization of the medium. The connectivity of these devices and location technologies also multiply the potential for local information, enabling a contextualized experience. This paper aims to analyse the adaptation of talk radio stations in Spain and Portugal to the radioapps from a content perspective and also to assess the possibilities of customizing access and consumption.

\section{Keywords}

Radioapps; Mobile radio; Online radio; Applications; Broadcasters; Smartphones; Mobile devices; Multimediality; Customization; Spain; Portugal.

Piñeiro-Otero, Teresa (2015). "Información, multimedialidad y personalización en las radioapps de España y Portugal". El profesional de la información, v. 24, n. 4, pp. 463-471.

http://dx.doi.org/10.3145/epi.2015.jul.13 


\section{Introducción}

En The feeling of power (1958) Asimov adelantaba una sociedad marcada por la existencia de ordenadores autónomos de bolsillo. Hoy estos dispositivos se han materializado y multiplicado dando lugar a un nuevo entorno social, móvil, abierto e interconectado (Weinberger, 2011). A nivel mundial los smartphones alcanzan ya al $72 \%$ de los usuarios móviles, en España al 84\% y en Portugal al 40\% (Ametic; Accenture, 2014; Anacom, 2014).

Las funciones de estos dispositivos trascienden el concepto de teléfono para constituir pantallas sociales, expansivas, envolventes y conectadas, donde convergen comunicación y contenido (Aguado; Feijóo; Martínez, 2013). Suponen una prolongación de la identidad del usuario debido a sus posibilidades de gestión autónoma y personalizada (Casseti; Sampietro, 2012). Su conectividad ha revolucionado el modo en que nos relacionamos con la información digital, lo que se ha reflejado en una cultura del acceso (Díaz-Noci, 2010; Meso-Ayerdi et al., 2014).

Para Scolari (2012) los smartphones se han convertido en grandes depredadores de atención, y han dado lugar a un usuario ávido de información continua, ubicua y personalizada (Nozal; González-Neira; Sanjuán, 2014). De este modo constituyen un soporte único para las nuevas manifestaciones mediáticas, entre ellas las radiofónicas (Aguado; Feijóo; Martínez, 2013).

\section{La radio móvil}

En este contexto la radio ha orientado su expansión a la telefonía móvil, una plataforma con la que ha experimentado en la última década. Desde Visual Radio (sistema de radio FM con datos para móvil puesto en marcha por Nokia en 2004), diversas iniciativas han explorado las posibilidades de la tecnología móvil para dotar a la radio de un lenguaje y expresividad propios (Cebrián-Herreros, 2008; Gallego-Pérez, 2012). No obstante hasta la normalización de las redes $3 \mathrm{G}$ el verdadero motor de la radio online móvil fue el podcasting (Sellas, 2014).

La tecnología de sindicación de audio, incorporada en 2004, conllevó la flexibilización del medio para una experiencia personalizada y la ampliación del universo de la radiofonía online a los dispositivos portátiles (Gallego-Pérez, 2012; Sellas, 2012). En la actualidad los smartphones y sus aplicaciones constituyen las principales plataformas de expansión de la radio móvil, por delante de otros dispositivos como las tablets (Piñeiro-Otero, 2014).

Radio y teléfono móvil comparten portabilidad y esencia sonora, características que les confieren un potencial único para el consumo multitasking o en movilidad (Fidalgo, 2013). Asimismo resulta reseñable su carácter íntimo y personal que incrementa sus posibilidades de engagement.

Más allá de sus posibilidades como medio síncrono y asíncrono, multimedia e interactivo, la radiofonía móvil incorpora nuevas oportunidades comunicativas. En los smartphones tiene la oportunidad de ofrecer contenidos geolocalizados, personalizados y participativos en una experiencia completa perfectamente imbricada (Videla-Rodríguez; Piñeiro-Otero,
2013). Para ello resulta necesario abordar el rediseño de los contenidos de la radio online (Cordeiro; Paulo, 2014).

La apropiación de esta nueva plataforma constituye para la radio una oportunidad única en atracción de oyentesusuarios (Rosales, 2013), también desde la perspectiva del acceso.

\section{En los smartphones la radio tiene la oportunidad de ofrecer contenidos geo- localizados, personalizados y participati- vos}

El creciente número de usuarios móviles conectados presenta un contexto propicio para la expansión de la radiofonía móvil en España y Portugal. Según IAB (2014), tres de cada cuatro internautas son usuarios de radio en internet, ya sea de versiones online de radios convencionales (internet-onlyradios) o servicios automatizados (Spotify, Grooveshark...). La mitad de estos usuarios disfrutan los contenidos radiofónicos a través de aplicaciones para dispositivos móviles.

Pese a la pluridimensionalidad de la radiofonía online, los usuarios continúan decantándose por las marcas convencionales de radio $(81,5 \%$ de internautas), aunque se incrementa la demanda de servicios automatizados (64,2\% de internautas) que remiten a una personalización del consumo sonoro. Precisamente la escucha "como y donde quiero" constituye la principal motivación para el uso de la radio online, esencial en el medio móvil (IAB, 2014).

Entre los principales hábitos de uso de la telefonía móvil, Xu et al. (2011) destacan una tendencia a la personalización de los smartphones. Ello se une al carácter personal de los terminales móviles en la construcción de una sono-esfera digital privada, con el mensaje como centro de la mediación tecnológica (Perona-Páez; Barbeito-Veloso; Fajula-Payet, 2014).

Este cambio en el paradigma comunicativo confiere a las emisoras generalistas un lugar privilegiado en la radiofonía móvil. La combinación de contenidos noticiosos y geolocalización en una única aplicación posibilita una experiencia más contextual y potencia la información local (Weiss, 2013). Dicha fórmula ha situado a las radioapps y newsapps o aplicaciones de noticias entre las más populares para smartphones (Xu et al., 2011).

Este estudio tiene por objeto analizar la adaptación de las radios generalistas de España y Portugal hacia la radio móvil, desde la perspectiva de los contenidos y la personalización de las aplicaciones, las radioapps. Dicho objeto se sitúa en la línea de los trabajos de Videla-Rodríguez y Piñeiro-Otero (2012; 2013), Piñeiro-Otero y Videla-Rodríguez (2013) y Ferreras-Rodríguez (2014) sobre las radioapps españolas; de Paulo (2013) y Cordeiro y Paulo (2014) relativos a la radiofórmula portuguesa, o de la aproximación plurinacional de Ramos y González-Molina (2014). Ahora bien, la concreción de este estudio en las radios generalistas ibéricas y su orientación hacia las oportunidades de personalización suponen un nuevo enfoque en el estudio de la radio móvil. 


\section{Materiales y métodos}

Para analizar la adaptación de las emisoras generalistas ibéricas a la radiofonía móvil se efectuó un estudio exploratorio de sus aplicaciones utilizando el análisis de contenido como método de investigación. Este método permitió examinar de forma sistemática procedimientos y rasgos formales de las radioapps.

La selección de la muestra de análisis se hizo por marcas radiofónicas y sistemas operativos. En el primer caso se seleccionaron las 7 emisoras generalistas de España y Portugal con mayor audiencia offline (EGM, febrero-noviembre 2014; Bareme Rádio, septiembre 2014): 4 españolas y 3 lusas.

Se acotó la muestra a los sistemas operativos iOS y Android, los de mayor penetración en el mercado ibérico. Se obtuvo una muestra de $7+7$ radioapps disponibles para ambos sistemas.

En las emisoras públicas, RNE y Antena1, resulta destacable el carácter colectivo de sus aplicaciones. La app de $R N E$ agrupa todas las radios de la corporación pública española mientras que Antena1 se integra en la aplicación de Rádio e Televisão de Portugal, hasta el punto de que resulta complejo delimitar su espacio y contenidos respecto a los de otros medios del ente.

Para analizar los contenidos y las posibilidades de personalización de las apps se elaboró una ficha de análisis en torno a tres parámetros:

1) Contenidos:

- presencia y uso de textos, imágenes, vídeos, hipervínculos;

- distribución de contenidos sonoros genera-

les y programas;

- contenidos más relevantes.

2) Personalización:

- diseño y organización de la app;

- selección emisora local-temática;

- playlist;

- suscripción y alertas;

- marcar para consumo posterior;

- otro

3) Geolocalización:

- autoselección de emisora;

- servicios geolocalizados;

- otros.

La recogida de datos se efectuó durante la semana del 20 al 26 de octubre de 2014.

\section{Una radioapp visual e informativa}

La relevancia de la información de actualidad y deportiva en las radios generalistas ibéricas se ha reflejado tanto en la estructura como en el lenguaje de sus aplicaciones.
En el diseño de su portada y principales niveles de navegación se decantan por maquetaciones en formato lista, que permiten a los usuarios una perspectiva instantánea de la información de actualidad y un acceso optimizado a las noticias de interés. La esencia colectiva de la aplicación de $R N E$ ha implicado un desplazamiento de su portada a niveles inferiores de navegación; sin embargo tanto la homepage genérica como la del espacio de la radio emplean un diseño en lista.

Únicamente las aplicaciones de COPE presentan una estructura alternativa al incorporar en la portada un diseño en parrilla, que ofrece una perspectiva global de la app, y relegar el formato lista a los siguientes niveles de navegación.

Pese a la convergencia en el diseño de las aplicaciones, el análisis de los contenidos ha permitido señalar dos diferencias según la nacionalidad:

- las emisoras portuguesas sitúan el listado de noticias de última hora como contenido principal en la portada, destacando en su maquetación las noticias más relevantes;

- las españolas trasladan este peso a la emisión en directo.

El protagonismo de la transmisión radiofónica en las
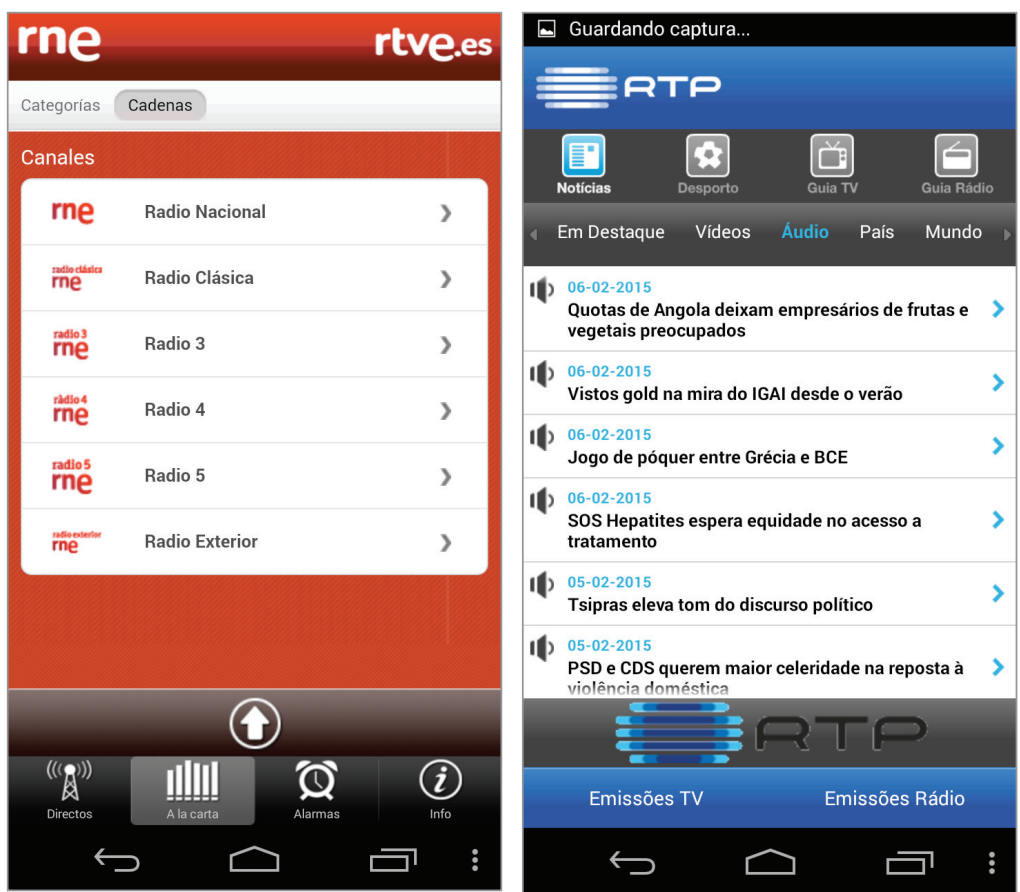

Imagen 1. Apps colectivas de RNE y de Antena1 (dentro de RTP). 


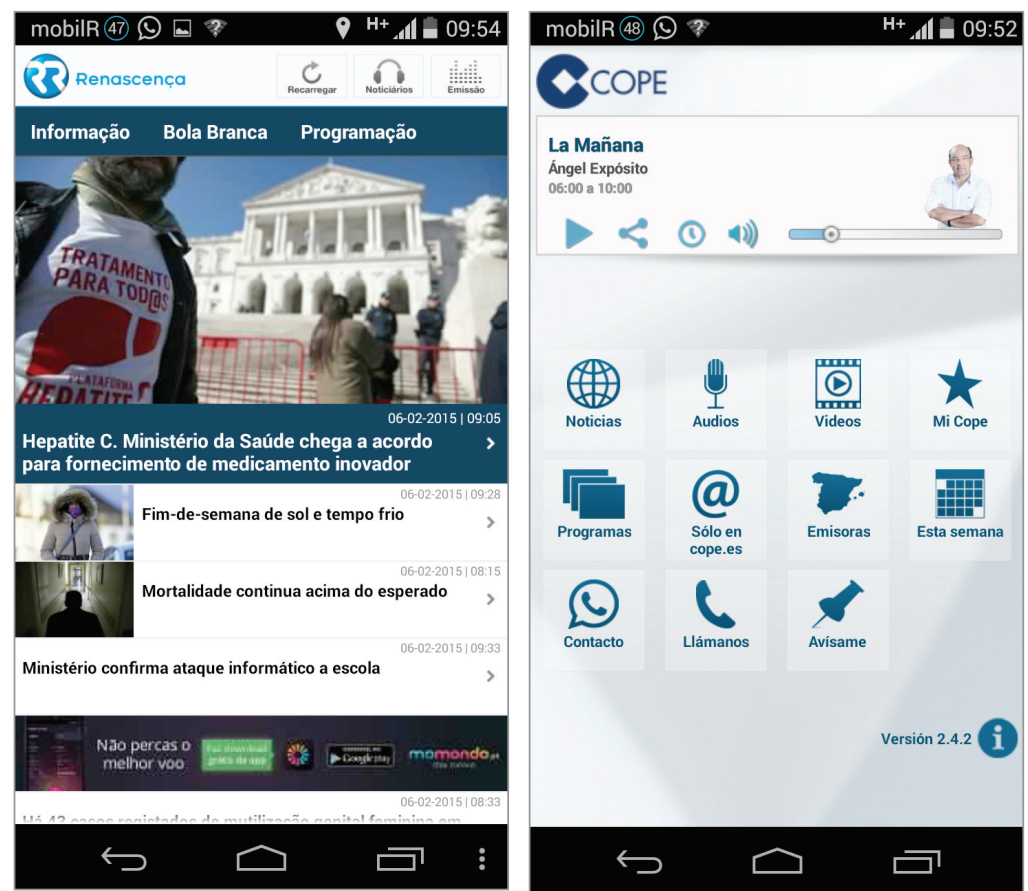

Imagen 2. Página de inicio con maquetación formato lista (Renascença) y parrilla (COPE).

TSF y Antena1 incorporan galerías audiovisuales a sus aplicaciones; unas galerías que -en el caso de RTP- se alimentan de los contenidos emitidos por la televisión pública.

Además de la galería audiovisual, TSF también utiliza ocasionalmente vídeos para acompañar a sus informaciones, un uso compartido por las aplicaciones de Renascença.

En la radio española el único ejemplo de integración audiovisual lo encontramos en COPE. Esta emisora vehicula en sus aplicaciones fragmentos de vídeo con una doble finalidad: ilustrar determinadas noticias y complementar los contenidos de sus principales programas.

\section{Emisión en directo y radio on demand}

Los contenidos sonoros, esencia de la radio convencional, se presentan en todas las radioapps analizadas combinando la emisión en directo con fórmulas de distribución on demand. Pese a ello se han comprobado al-

radioapps españolas puede ser total, pasando los titulares de última hora a otras pestañas o subniveles de navegación (Onda Cero, RNE y COPE) o compartir presencia con la información de actualidad en la portada (SER).

La relevancia de la información de actualidad ha derivado en una mayor presencia de contenidos textuales. Todas las emisoras privadas ofrecen a sus usuarios las noticias de actualidad en formato texto, habitualmente acompañadas de imágenes y/o audios. En las radios públicas, Antena1 y RNE, el peso del contenido textual es menor, limitándose a titulares y/o resúmenes informativos.

Las radioapps portuguesas dan prioridad a la información de actualidad en formato textual y las españolas a la emisión en directo

El estudio de los contenidos ha permitido constatar la tendencia en las radioapps portuguesas a complementar información textual e imagen siguiendo las pautas de las aplicaciones de prensa para smartphone.

La influencia de las newsapps en la radiofonía móvil lusa también se refleja en la utilización de hipervínculos. TSF y Renascença -las dos emisoras privadas analizadas- utilizan links para vincular informaciones relacionadas, posibilitando diversos niveles de lectura, siempre dentro de la propia aplicación.

De las españolas, sólo COPE y RNE incorporan hipervínculos en sus contenidos. Esta inclusión permite expandir la experiencia del usuario con el acceso al universo de canales de la radio online desde la propia aplicación.

Otros contenidos como los audiovisuales cuentan con una presencia limitada. De las emisoras analizadas únicamente gunas divergencias en el tipo de acceso y en la utilización de estos contenidos sonoros.

La prevalencia de la emisión en directo en las apps españolas se ha reflejado en el acceso previsto. Todas las cadenas españolas permiten disfrutar de la programación en antena desde que se ingresa en la aplicación. Excepcionalmente la $S E R$ exige al usuario configurar una emisora de preferencia en su primer ingreso, permitiendo la personalización de la señal recibida.

En las aplicaciones portuguesas la emisión en directo se activa siempre a demanda del usuario, siendo precisa su conexión mediante un botón situado en el menú o en la barra de acción.

En los contenidos en diferido el streaming se impone sobre otras fórmulas de distribución on demand. Todas las emisoras generalistas incorporan contenidos en streaming tanto en la información de actualidad y deportiva como en sus principales programas. No obstante, mientras las españolas integran en el apartado de información de actualidad fragmentos de audio, como contenido autónomo o complemento de un texto, las portuguesas limitan el uso del streaming en esta sección a la publicación del último boletín horario.

\section{Los contenidos sonoros se presentan combinando la emisión en directo con fórmulas de distribución on demand}

Esta divergencia entre las aplicaciones de España y Portugal, derivada de su diferente concepción y organización de la información de actualidad, queda neutralizada en el servicio de programas a la carta.

Todas las aplicaciones analizadas prevén el acceso y consu- 

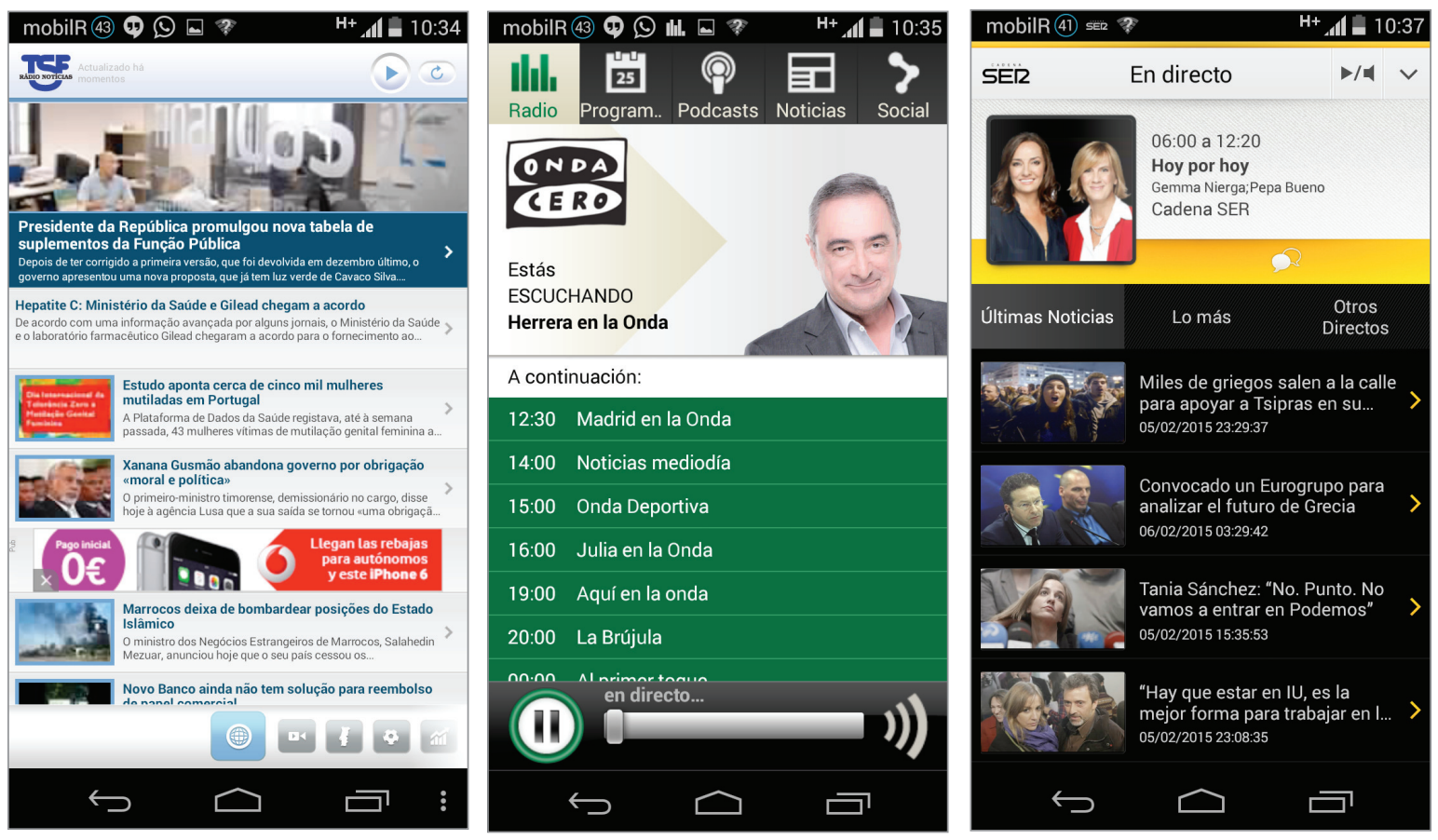

Imagen 3. Homepages formato lista con mayor protagonismo de información de actualidad (TSF), emisión en directo (Onda Cero), y con equilibrio de dichos contenidos (SER).

mo de sus principales programas on demand aunque se señalan diversas tendencias en su distribución.

Antena1 y TSF vehiculan en las apps sus principales programas fragmentados, al contrario que Renascença que se decanta por la distribución de dichos contenidos íntegros.

Por su parte, las emisoras españolas optan por una vía intermedia: la fragmentación de los principales programas en unidades de contenido -atendiendo a su estructura, género, temática o duración- y la distribución del programa completo en aquellos espacios minoritarios o de menor duración.

\section{La fragmentación de contenidos supone un avance en la adaptación a la platafor- ma móvil así como una consecución de un medio más personal}

Onda Cero también incorpora esta doble posibilidad a sus principales programas, de modo que el oyente-usuario puede acceder a estos espacios fragmentados en unidades de contenido o consumirlos íntegros.

Más allá de las diferencias en la distribución de los contenidos on demand, las radioapps españolas y portuguesas también presentan divergencias en la fragmentación de sus principales programas. Mientras Antena1 y TSF segmentan sus programas en bloques homogéneos, atendiendo exclusivamente a su duración, las radioapps españolas combinan este criterio de fragmentación con el de contenido.

$R N E$, Onda Cero, SER y COPE distribuyen sus programas en bloques temporales, entrevistas, microespacios u otro tipo de segmentos temáticos. A pesar de que la subdivisión de las emisiones radiofónicas atendiendo a su extensión continúa siendo la más habitual en la muestra, el análisis efectuado ha permitido esbozar algunas tendencias de fragmentación en función del tipo de programa.

Si nos centramos en los seis espacios de mayor entidad en las parrillas de la radio española -programas informativos matinales, magazines de tarde, informativos nocturnos, deportivos laborables, de retransmisión deportiva y matinales fin de semana- se puede señalar una fórmula de distribución de contenidos común a todos ellos: separando bloques temporales y entrevistas.

En los programas informativos matinales esta segmentación puede enriquecerse con la separación de otros géneros radiofónicos como el comentario (Hoy por hoy, Herrera en la Onda), tertulia o participación del oyente (Herrera en la onda). La fragmentación de contenidos atendiendo al género es común a los informativos nocturnos donde, además del comentario (Hora 25, La brújula) y la tertulia (La linterna, 24), se introducen otros criterios de segmentación como el temático (Hora 25, La brújula, 24).

Este tipo de segmentación por apartados temáticos o microespacios es más frecuente en algunos programas matinales de fin de semana ( $A$ vivir que son dos días, Te doy mi palabra), cuya orientación hacia contenidos culturales y lúdicos les confiere una estructura más heterogénea que los programas de información de actualidad.

Del mismo modo sucede con la distribución de los magazines vespertinos, entre los que destaca Julia en la Onda por presentar su emisión íntegra y el programa fragmentado exclusivamente por contenidos: entrevista, comentarios y apartados temáticos de cada emisión del programa.

En la programación deportiva se puede diferenciar entre los 
Tabla 2. Distribución de contenidos en los principales programas por tipo y emisora

\begin{tabular}{|c|c|c|c|c|c|c|c|c|c|}
\hline & & \multirow[b]{2}{*}{ Íntegro } & \multicolumn{7}{|c|}{ Fragmentación de los contenidos } \\
\hline & & & Bloques & Entrevista & Tertulia & Comentario & $\begin{array}{l}\text { Apartado } \\
\text { temático }\end{array}$ & Microespacio & Goles \\
\hline \multicolumn{10}{|c|}{ Programas informativos matinales } \\
\hline Hoy por hoy & SER & & * & * & & * & & & \\
\hline $\begin{array}{l}\text { Herrera en la } \\
\text { Onda }\end{array}$ & $\begin{array}{l}\text { Onda } \\
\text { Cero }\end{array}$ & & * & * & * & * & & * & \\
\hline La mañana & COPE & & * & * & & & & & \\
\hline Las mañanas & $R N E$ & & * & * & & & & & \\
\hline \multicolumn{10}{|l|}{ Magazines tarde } \\
\hline La ventana & SER & & * & * & & & * & * & \\
\hline Julia en la Onda & $\begin{array}{l}\text { Onda } \\
\text { Cero }\end{array}$ & * & & * & & * & * & & \\
\hline La tarde & COPE & & * & & & & & & \\
\hline Esto me suena & $R N E$ & & * & * & & & & & \\
\hline \multicolumn{10}{|c|}{ Informativos noche } \\
\hline Hora 25 & SER & & * & * & & * & * & * & \\
\hline La brújula & $\begin{array}{l}\text { Onda } \\
\text { Cero }\end{array}$ & & * & * & & $*$ & * & * & \\
\hline La linterna & COPE & & * & & * & & & & \\
\hline 24 & $R N E$ & & & * & * & & $x$ & & \\
\hline \multicolumn{10}{|c|}{ Retransmisión deportiva } \\
\hline Carrusel & SER & & $*$ & & & & * & $*$ & * \\
\hline Tiempo de juego & COPE & & $*$ & * & & & & & * \\
\hline Radio estadio & $\begin{array}{l}\text { Onda } \\
\text { Cero }\end{array}$ & & $*$ & * & & $*$ & * & & * \\
\hline Tablero deportivo & $R N E$ & & * & & & & & & \\
\hline \multicolumn{10}{|c|}{ Deportes laborables } \\
\hline El larguero & SER & & * & * & & & * & & \\
\hline $\begin{array}{l}\text { El partido de } \\
\text { las } 12\end{array}$ & COPE & & * & * & & & & & \\
\hline Al primer toque & $\begin{array}{l}\text { Onda } \\
\text { Cero }\end{array}$ & * & & * & & & * & & \\
\hline $\begin{array}{l}\text { Radiogaceta de } \\
\text { los deportes }\end{array}$ & $R N E$ & * & & & & & & & \\
\hline \multicolumn{10}{|c|}{ Matinales fin de semana } \\
\hline $\begin{array}{l}\text { A vivir que son } \\
\text { dos días }\end{array}$ & SER & & * & * & & & * & & \\
\hline $\begin{array}{l}\text { No es un día } \\
\text { cualquiera }\end{array}$ & RNE1 & & * & * & & & & & \\
\hline Te doy mi palabra & $\begin{array}{l}\text { Onda } \\
\text { Cero }\end{array}$ & & * & * & & & * & & \\
\hline $\begin{array}{l}\text { La mañana fin de } \\
\text { semana }\end{array}$ & COPE & & * & & & & & & \\
\hline
\end{tabular}

espacios emitidos en días laborables, que siguen las pautas de los magazines vespertinos, y las retransmisiones deportivas, que presentan un sistema de distribución característico. En las apps de las cadenas privadas estos espacios permiten consumir de forma individual cada uno de los goles de la jornada, además de otros contenidos divididos por tipo de deporte, comentario (Radio estadio) o microespacios (Carrusel deportivo).

El estudio de la distribución de los principales programas en las apps españolas ha puesto de manifiesto una mayor adaptación de Onda Cero para el consumo on demand. Todos los contenidos analizados permiten dos formas de consumo:

- la escucha continua de archivos íntegros (o divididos en dos-tres bloques temporales consecutivos), próxima a la radio convencional;

- el acceso no lineal a contenidos concretos de interés: entrevista, tertulia, apartado temático, etc.

Esta apuesta por la fragmentación es un avance en la adaptación de la radio a la plataforma móvil y a sus tendencias de 
consumo, al tiempo que implica un paso más en la consecución de un medio más personal.

Frente a la multiplicidad de contenidos en streaming, los podcasts -considerados el formato estrella de la radio en la era de la portabilidad- apenas tienen presencia en la actualidad en la muestra de estudio. Sólo Renascença incorpora contenidos en este formato.

No obstante, la identificación del podcasting con el consumo bajo demanda ha propiciado que se etiqueten como tal otros archivos sonoros que, aún permitiendo su disfrute online y su descarga para un consumo offline, no son contenidos sindicados (característica esencial en los podcasts).

\section{Posibilidades de personalización}

La incorporación de contenidos on demand en las radioapps ibéricas supone un avance en la personalización del medio. Más allá de esta opción, las emisoras analizadas apenas han incorporado otras posibilidades en sus aplicaciones móviles.

Desde la perspectiva del diseño y organización de la aplicación tan sólo las apps de Renascença y TSF para iPhone permiten a sus usuarios modificar determinados contenidos del menú. Se trata de una opción básica de personalización no prevista en su versión Android.

Del mismo modo la mayoría de las radioapps generalistas no permiten la selección de una emisora concreta -local o especializada- para el acceso a sus contenidos.

El sistema español de radiodifusión en cadena, basado en una red de emisoras locales, otorga mayor importancia a la información de proximidad que las principales radios portuguesas. Esta esencia local cuenta con un limitado reflejo en las apps analizadas. Solamente las aplicaciones de COPE y $S E R$ prevén la selección manual de una emisora local, una función que no se ha implementado para todas las estaciones de estas cadenas. La SER también permite la selección de emisora por proximidad geográfica, basándose en la tecnología de geolocalización del smartphone.

En lo que se refiere a la elección de radio por temática, aunque la mayoría de la muestra pertenece a grupos multimedia titulares de diversas marcas radiofónicas (con la excepción de TSF), sólo RNE y Antena1 brindan a sus usuarios la posibilidad de seleccionar otra emisora (especializada) sin salir de su aplicación. El carácter colectivo de dichas apps facilita el acceso a los contenidos-directo y on demand-de las emisoras que forman parte de ambos entes públicos de radiotelevisión.

En la selección y organización de los contenidos de interés para su posterior consumo, se vuelve a poner de manifiesto la limitada utilización de herramientas que facilitan la creación de una radio personal. Ninguna de las apps analizadas permite la creación de playlists de contenidos y la inclusión de otras funciones, como suscripciones a programas, secciones de interés o alertas, es todavía minoritaria. Sólo las aplicaciones de COPE (iOS y Android) y Antena1 para iPhone permiten a sus usuarios la suscripción a contenidos radiofónicos que conformarán su área personal. Estas aplicaciones, a las que se suma TSF, también prevén la activación de alertas informativas que permiten recibir notificaciones automáticas en el móvil cada vez que se produce un acontecimiento de actualidad relevante.

La integración de estas funciones en las radioapps, además de suponer un avance hacia un medio más personalizado, facilita el acceso y disfrute de los contenidos de interés. Alertas y sobre todo suscripciones, se consideran push technologies en tanto que brindan a los usuarios las últimas actualizaciones de su selección de forma automática.

Otra función interesante desde la perspectiva de la personalización del medio móvil es la de postergar el consumo. Los usuarios de Onda Cero y Renascença pueden marcar en sus aplicaciones aquellos contenidos de interés para su consumo en otro momento o lugar. Este proceso facilita la recuperación de dichos contenidos a través de un apartado concreto dentro del menú.

Si bien la tecnología de geolocalización es una oportunidad para la información local u otro tipo de servicios contextuales como el tiempo o el tráfico, ya presentes en la radio convencional, las radioapps ibéricas todavía no han explotado sus posibilidades. Más allá de la selección de emisora prevista por las apps de la $S E R$, se ha comprobado otra función de esta tecnología en la aplicación de COPE para iPhone. En este caso la geolocalización se integra en la app de Tiempo de juego (el programa de retransmisión deportiva de la cadena) y permite a los individuos -debidamente registrados- interactuar con otros geográficamente próximos. Esto constituye un paso más en la conexión y cohesión de la comunidad de oyentes-usuarios del programa.

\section{Alertas y suscripciones funcionan como push technologies en tanto que brindan a los usuarios las últimas actualizaciones de su selección de forma automática}

\section{Conclusiones}

Las radios generalistas de España y Portugal han ampliado su universo online a las plataformas móviles con la oferta de diversas aplicaciones para los principales sistemas operativos. Pese a esta oferta, el análisis de la estructura, contenidos y posibilidades de personalización revela que las radioapps todavía se encuentran en un proceso de adaptación y apropiación de esta plataforma.

En la implementación de sus aplicaciones estas radios han seguido las pautas de las newsapps, tanto en apariencia y funciones, como en la relevancia que adquiere la información de actualidad en formato textual complementada con otros elementos multimedia. Esta proximidad resulta más patente en las radioapps portuguesas, donde los contenidos informativos priman sobre la emisión en directo y las noticias son esencialmente visuales.

Las emisoras generalistas también han apostado por flexibilizar la experiencia de usuario con la distribución de sus principales programas on demand, fragmentándolos para un consumo optimizado desde el smartphone. Las aplicaciones de radios españolas amplían esta posibilidad a la in- 
formación de actualidad con la incorporación de archivos de audio en streaming, como noticias o como complemento de éstas.

La previsión por parte de las radioapps analizadas de un consumo síncrono y asíncrono del medio subraya la evolución de la radiofonía móvil respecto a los estudios de Videla-Rodríguez y Piñeiro-Otero (2012, 2013), evolución en la que los podcasts han quedado relegados respecto a la distribución de contenidos en streaming.

Si, como señala Sellas (2012), la radio generalista camina hacia nuevas formas de segmentación y distribución de contenidos que simplifiquen su acceso y uso, en la plataforma móvil el streaming cuenta con más potencialidades que el podcast, especialmente si prevé alguna fórmula de suscripción.

La evolución vivida por las radioapps respecto a los primeros estudios sobre la radio móvil española (Videla-Rodríguez; Piñeiro-Otero, 2012) y portuguesa (Paulo, 2013) refleja un avance hacia un medio más personal.

Si bien las posibilidades de personalización que brindan las aplicaciones analizadas son todavía muy limitadas, en la línea señalada por los estudios de Ferreras-Rodríguez (2014) o Cordeiro y Paulo (2014), la fragmentación de los principales programas supone un avance en la creación de una radio a medida. En este sentido se han podido esbozar diversas tendencias en la distribución de los contenidos en las apps por tipo de programa; contenidos que adquieren mayor entidad como unidades de sentido completo y que van a permitir flexibilizar al máximo la experiencia del usuario.

No obstante, las radioapps generalistas muestran todavía un uso limitado de aspectos como la hipertextualidad y la geolocalización, tanto en lo que respecta a sus contenidos como a su distribución. Esta carencia, ya destacada por Videla-Rodríguez y Piñeiro-Otero (2012, 2013) y Ramos y González-Molina (2014) resulta común a todos los cibermedios móviles en el contexto actual, como destacan MesoAyerdi et al. (2014).

Aunque los smartphones constituyen una oportunidad para un consumo más personal y contextual del medio radiofónico, las emisoras analizadas todavía no han logrado apropiarse de esta plataforma de difusión. En la consecución de un lenguaje y expresividad propios, las radioapps españolas han abogado por rescatar la esencia sonora del medio, mientras que las portuguesas se han decantado por una apuesta más visual con predominio del contenido textual, en la línea de las newsapps.

El estudio nos lleva a coincidir con Ferreras-Rodríguez (2014) en que, más que un nuevo medio con posibilidades y expresividad propias, las emisoras generalistas todavía perciben el móvil como un soporte de la radio convencional de modo que no conciben sus contenidos como productos autónomos e independientes.

La cuestión que subyace es si estamos caminando hacia un nuevo concepto de radio, como afirman Cordeiro y Paulo (2014), o resulta más preciso referirnos a marcas radiofónicas con acceso desde el móvil en lugar de radioapps propiamente dichas.

\section{Bibliografía}

Aguado, Juan-Miguel; Feijóo, Claudio; Martínez, Inmaculada (2013). La comunicación móvil hacia un nuevo ecosistema digital. Barcelona: Gedisa. ISBN: 9788497847827

Ametic; Acenture (2014). Somos digitales 2014. $h t t p: / / g o o . g l / s A O P G z$

Anacom (2014). Serviços móveis. Informação estatística. 2o trimestre 2014.

http://goo.gl/uQutQ5

Casseti, Francesco; Sampietro, Sara (2012). "With eyes, with hands. The relocation of cinema into the iPhone". En: Snickars, Pelle; Vonderay, Patrick (eds.). Moving data. The iPhone and the future of media. New York: Columbia University Press. pp. 19-32. ISBN: 9780231504386

Cebrián-Herreros, Mariano (2008). "Radio y convergencia tecnológica en Europa. Expansión de los cibermedios fijos y móviles". En: Ortega-Carmona, Leopoldo. 7a Bienal internacional de Radio México. Colonia del Valle: Radio Educación. pp. 69-88.

http://goo.gl/WDa3Zp

Cordeiro, Paula; Paulo, Nádia (2014). "A rádio numa app: Convergência multimédia e os conteúdos da rádio". Media \& jornalismo, v. 24, n. 13, pp. 117-133.

http://goo.gl/ceNulL

Díaz-Noci, Javier (2010). “Medios de comunicación en internet: algunas tendencias". El profesional de la información, v. 19, n. 6 , pp. 561-567.

http://www.elprofesionaldelainformacion.com/contenidos/2010/ noviembre/medios_comunicacion.pdf http://dx.doi.org/10.3145/epi.2010.nov.01

Ferreras-Rodríguez, Eva (2014). "Contenidos de las aplicaciones de las radios autonómicas para dispositivos móviles. En: IV Congreso internacional de la Asociación Española de Investigación de la Comunicación AE-IC. Bilbao: AE-IC y Universidad del País Vasco, pp. 1456-1470.

http://www.aeic2014bilbao.org/download/aeic2014bilbao_ comunicaciones.pdf

Fidalgo, António (2013). “O celular como rádio de pilhas na era da internet. Rádio IP no celular". En: Barbosa, Suzana; Mielniczuk, Luciana. Jornalismo e technologias móveis. Covilha: Labcom, pp. 11-24. ISBN: 9789896541019 http://www.livros/abcom.ubi.pt/book/98

Gallego-Pérez, Ignacio (2012). "La distribución de contenidos de audio. Relaciones entre podcasting, radio y movilidad". Telos, n. 92.

http://goo.gl/7pWrVZ

IAB (2014). Primer estudio de la radio online. http://goo.gl/q1TqLo

Meso-Ayerdi, Koldobika; Larrondo-Ureta, Ainara; PeñaFernández, Simón; Rivero-Santamarina, Diana (2014). "Audiencias activas en el ecosistema móvil. Análisis de las opciones de interacción de los usuarios en los cibermedios españoles a través de la web, los teléfonos móviles y las tabletas". Hipertext.net, n. 12.

http://dx.doi.org/10.2436/20.8050.01.9 
Nozal, Teresa; González-Neira, Ana; Sanjuán, Antonio (2014). "Análisis de ediciones vespertinas de prensa para tablets". El profesional de la información, v. 23, n. 4, pp. 393400.

\section{http://dx.doi.org/10.3145/epi.2014.jul.07}

Paulo, Nadia (2013). A convergência multimédia e os conteúdos móveis da rádio. Tesis de master. Lisboa: Iscsp. http://www.repository.utl.pt/handle/10400.5/6498

Perona-Páez, Juan-José; Barbeito-Veloso, María-Luz; Fajula-Payet, Anna (2014). "Los jóvenes ante la sono-esfera digital: medios, dispositivos y hábitos de consumo sonoro". Comunicación y sociedad, v. 27, n. 1, pp. 205-224.

http://www.unav.es/fcom/communication-society/es/ resumen.php?art_id $=487$

Piñeiro-Otero, Teresa (2014). "La publicidad en las radioapps ibéricas. Falta de definición de un soporte publicitario sin límites". International journal of marketing, communication and new media, v. 2, n. 3, pp. 38-56.

http://u3isjournal.isvouga.pt/index.php/ijmcnm/article/ view/51

Piñeiro-Otero, Teresa; Videla-Rodríguez, José-Juan (2013). "La participación de los oyentes en las radioapps españolas. Prácticas convencionales en la era de la portabilidad". AdComunica, n. 5, pp. 67-89.

http://www.adcomunicarevista.com/ojs/index.php/ adcomunica/article/view/88

Ramos, Fátima; González-Molina, Sonia (2014). “La radio móvil en Europa y en Estados Unidos: un análisis comparativo del uso periodístico de las apps de las principales emisoras en los smartphones". En: Sierra, Javier; García-García, Francisco. Tecnología y narrativa audiovisual. Madrid: Fragua, pp. 169-192. ISBN: 9788470746277

Rosales, Rey G. (2013). "Citizen participation and the uses of mobile technology in radio broadcasting". Telematics and informatics, v. 30, n. 3, pp. 252-257.

http://dx.doi.org/10.1016/j.tele.2012.04.006

Sellas, Toni (2012). "A two-dimensional approach to the study of podcasting in Spanish talk radio stations". The radio journal, v. 10, n. 1, pp. 7-22.

http://goo.gl//p2HsW

http://dx.doi.org/10.1386/rjao.10.1.7_1

Scolari, Carlos (2012). "Comunicación digital. Recuerdos del futuro". El profesional de la información, v. 21, n. 4, pp. 337340.

http://dx.doi.org/10.3145/epi.2012.jul.01

Videla-Rodríguez, José-Juan; Piñeiro-Otero, Teresa (2012). "Apps de radio. Movilidad para un medio radiofónico sin límites". Prisma, v. 17.

http://revistas.ua.pt/index.php/prismacom/article/ view/2020

Videla-Rodríguez, José-Juan; Piñeiro-Otero, Teresa (2013). "La radio móvil en España. Tendencias actuales en las apps para dispositivos móviles". Palabra clave, v. 16, n. 1, pp. 129153.

http://www.redalyc.org/articulo.oa?id=64926739006

Weinberger, David (2011). Too big to know. New York: Basic Books. ISBN: 9780465021420

Weiss, Amy-Schmitz (2013). "Exploring news apps and location-based services on the smartphone". Journalism \& mass communication quarterly, v. 90, n. 3, pp. 435-456.

http://dx.doi.org/10.1177/1077699013493788

Xu, Qiang; Erman, Jefrey; Gerber, Alexandre; Mao, Morley; Pang, Jefrey; Venkataraman, Shobha (2011). "Identifying diverse usage behaviors of smartphone apps". En: IMC'11. https://web.eecs.umich.edu/ zmao/Papers/imc11_xu.pdf

\section{Decir la ciencia. Divulgación y periodismo de Galileo a Twitter}

Vladimir de Semir, director académico del Máster en Comunicación Científica del UPF-IDEC.

\section{Decir la ciencia}

Divulgación y periodismo cientifico de Galileo a Twitter

Vladimir de Semir

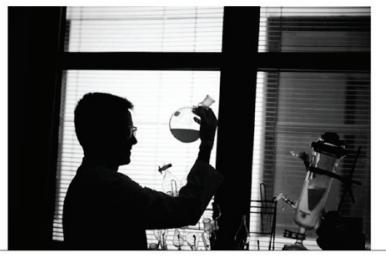

Ensayo sobre las relaciones entre el periodismo y la ciencia, y sobre su impacto en la sociedad del conocimiento. Reflexión sobre la importancia que ha tenido a lo largo de la historia la divulgación de la información y el saber en el desarrollo del conocimiento.

Vladimir de Semir piensa que, en esta época de máxima accesibilidad a la cultura y al saber gracias a las tecnologías de la información y de la comunicación, la tarea del periodismo divulgativo es más necesaria que nunca. 'En medio de una auténtica marea informativa, sólo los buenos profesionales dotados de adecuado espíritu crítico son capaces de informar con rigor, distinguiendo la noticia del rumor, el hecho contrastado de la creencia, la mentira interesada de la certeza relativa en la que se sientan las bases del progreso y el conocimiento'.

El libro, dividido en catorce capítulos, es una radiografía de la profesión. El autor va desgranando las consideraciones aprendidas durante más de cuarenta años de trayectoria profesional - «una época dorada del periodismo científico», como él la califica y nos ofrece análisis y reflexiones que sintetizan la historia del oficio y proponen una visión de cómo debería ser en el futuro.

De Semir, Vladimir (2015). Decir la ciencia. Divulgación y periodismo de Galileo a Twitter. Barcelona: Universitat de Barcelona, 324 pp. ISBN: 9788447539079 PVP: $25 €$ 